\title{
A Technique for the Measurement of Visceral Fat by Ultrasonography: Comparison of Measurements by Ultrasonography and Computed Tomography
}

\author{
Masashi Hirooka, Teru Kumagi, Kiyotaka Kurose, Seiji NaKAnishi, \\ Kojiro MichitaKA*, Bunzo MAtsuURA, Norio HoRIIKE and Morikazu ONJI
}

\begin{abstract}
Objective This study aims to create a method of calculating intra-abdominal visceral fat volume by using ultrasound (US). The visceral fat volume measured by US was evaluated by comparison with the volume measured by computed tomography (CT).

Methods Eighty-seven patients $(52$ males and 35 females) were enrolled in this study. Both US and CT were performed, and the visceral fat volume was measured. Both the distance and thickness of the parameters in US were measured as follows: 1) the distance between the internal surface of the abdominal muscle and the splenic vein, 2) the distance between the internal surface of the abdominal muscle and the posterior wall of aorta on the umbilicus, and 3) the thickness of the fat layer of the posterior right renal wall.

Results The equation was calculated as follows: [visceral fat volume] $=-9.008+1.191 \times[$ distance between the internal surface of the abdominal muscle and the splenic vein $(\mathrm{mm})]+0.987 \times[$ distance between the internal surface of the abdominal muscle and the posterior wall of the aorta on the umbilicus $(\mathrm{mm})]+3.644 \times[$ thickness of the fat layer of the posterior right renal wall $(\mathrm{mm})]$. There was a good correlation between the visceral fat volume calculated by the above equation and the volume by $\mathrm{CT}$ described $(r=0.860, p<0.0001)$.

Conclusion The measurement of the visceral fat volume using US provided results as effectively as CT, and it was proven to be a useful method.

(Internal Medicine 44: 794-799, 2005)
\end{abstract}

Key words: visceral fat volume, ultrasonography

\section{Introduction}

Obesity is an important risk factor in diabetes mellitus, hypertension, hyperlipidemia, and cardiovascular diseases. Recently, metabolic syndrome has been suggested as being an accumulation of several conditions, such as glucose intolerance, hypertension, and hyperlipidemia in individual patients. There have been a number of reports on this syndrome that indicate a relation with insulin resistance, which causes syndrome X (insulin resistance, impaired glucose tolerance, hyperinsulinemia, increase in low-density lipoprotein, low high-density lipoprotein, and hypertension) (1), the deadly quartet (upper-body obesity, glucose intolerance, hypertriglyceridemia, and hypertension) (2), and insulin resistance syndrome (obesity, non-insulin-dependent diabetes mellitus, hyperinsulinemia, hyperlipidemia, and coronary artery disease) (3).

Moreover, nowadays, the distribution disorder of fatty tissue, especially with the higher accumulation of visceral fat among the various kinds of obesity, complicates metabolic disorders; this has been reported as visceral fat syndrome (visceral fat accumulation, glucose intolerance, hyperlipidemia, and hypertension) (4-6).

Evaluation of visceral fat has been conducted using waistto-hip circumference ratio $(7,8)$ and several images using CT (9-12), magnetic resonance imaging (MRI) (13-17), or US (18-22). Of these methods, evaluation using CT is the most useful and popular method. However, CT exposes the patient to ionizing radiation, and MRI is much more expensive than other methods.

This study aims to create a novel method of easily calculating intra-abdominal visceral fat volume by using US, which does not expose the patient to ionizing radiation. The visceral fat volume measured by US was evaluated by comparison with the volume measured by $\mathrm{CT}$.

From the Third Department of Internal Medicine and *the Endoscopy Center, Ehime University School of Medicine, Ehime

Received for publication April 9, 2004; Accepted for publication May 11, 2005

Reprint requests should be addressed to Dr. Morikazu Onji, the Third Department of Internal Medicine, Ehime University School of Medicine, Shizukawa, Touon, Ehime 791-0295 


\section{Materials and Method}

\section{Patients}

Eighty-seven patients (52 males and 35 females) who were admitted to our department between June 2002 and June 2003 were enrolled in this study. The mean age was $54.9 \pm 16.7$ years. The mean BMI was $25.3 \pm 2.92$. Of these patients, 38 had chronic liver disease, 29 had gastrointestinal disease, 10 had diabetes mellitus, 5 had gall bladder stone, 3 had common bile duct stone, and 2 had thyroid disease. Patients with a history of abdominal operation, ascites, malignant disease, and severe hepatic failure were excluded from this study. US and CT were performed in these patients for evaluating visceral fat.

Moreover in order to confirm reproduction by well experienced sonographers in abdominal ultrasound, 5 healthy volunteers (mean BMI: 23.2 \pm 3.64 ) were enrolled. These volunteers underwent US only in order to confirm reproduction of US. The mean age was $28.0 \pm 2.91$ years, and they had no disease. We obtained informed consent from all patients.

\section{Biochemical study}

The height $(\mathrm{cm})$ and weight $(\mathrm{kg})$ of each patient was measured, and body mass index (BMI, $\mathrm{kg} / \mathrm{cm}^{2}$ ) was calculated. Asparate aminotransferase (AST), alanine aminotransferase (ALT), fasting plasma glucose, uric acid, total cholesterol, triglyceride, high-density lipoprotein (HDL) cholesterol, and leptin in sera were measured. The 75-g oral glucose tolerance test was performed in all patients. The 36 patients with a BMI of more than 25 were divided into 2 groups; the non-visceral fat type (subcutaneous fat type; $\mathrm{n}=12)$, and the visceral fat type $(\mathrm{n}=24)$. By calculating the fat volume used for these patients by US, visceral fat type was considered as more than $100 \mathrm{~cm}^{2}$ and non-visceral fat type was less than $100 \mathrm{~cm}^{2}$. Height, weight, and BMI were expressed as mean \pm SD. The biochemical markers described above were also compared among these 2 groups.

\section{Ultrasonography}

Within 3 days, US (SSD-6500 ultrasound machine, ALOKA, Tokyo, Japan) and CT were performed after an overnight fast, and the visceral fat volume was measured in the supine position at the end of a normal exhalation. Both the distance and the thickness of the parameters were measured as follows: 1) the distance between the internal surface of the abdominal muscle and the splenic vein (Fig. 1a), 2) the distance between the internal surface of the abdominal muscle and the posterior wall of the aorta on the umbilicus (Fig. $1 b), 3$ ) thickness of the fat layer of the posterior right renal wall in the right posterior perinephric space (Fig. 1c), and 4) the thickness of both the subcutaneous and preperitoneal fat layers in the xiphoid process (Fig. 1d). A 3.5-MHz convexarray probe was used to measure each parameter, other than the thickness of both the subcutaneous and the preperitoneal fat layers at the xiphoid process by performing a longitudinal scan, which was measured using a $7.5-\mathrm{MHz}$ linear-array
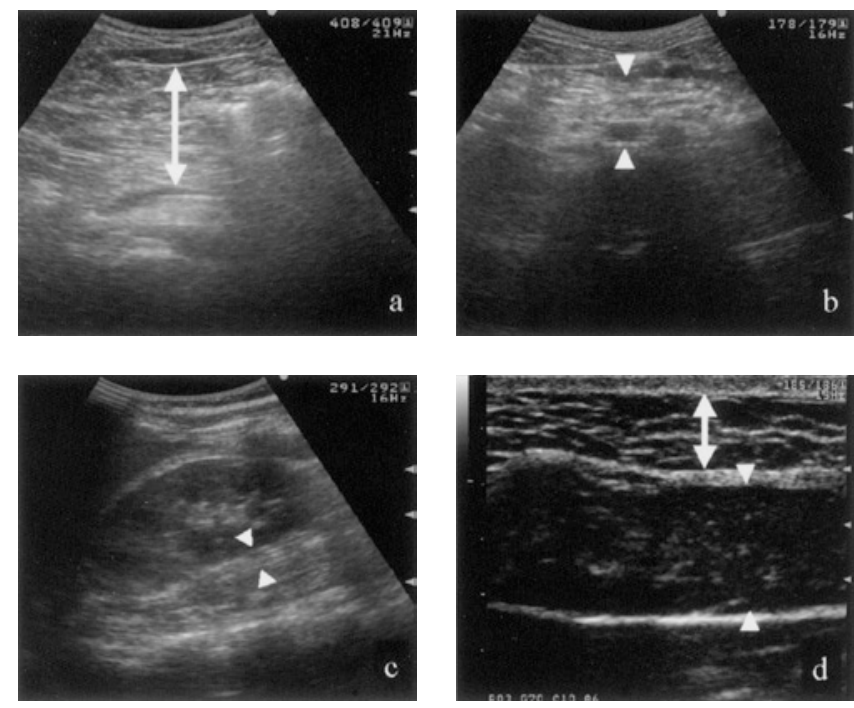

Figure 1. Parameter for visceral fat measured by ultrasonography. Both the distance and the thickness of the parameters were measured: a) the distance between the internal surface of abdominal muscle and splenic vein, b) the distance between the internal surface of the abdominal muscle and the posterior wall of the aorta on the umbilicus, c) the thickness of the fat layer of the posterior right renal wall, d) the thickness of both the subcutaneous and preperitoneal fat layers in the xiphoid process.

probe. The transducer was placed vertically against the skin as lightly as possible to prevent compression of the fat layers. The distance between the internal surface of the abdominal muscle and the splenic vein was scanned transversely in the midline. When the splenic vein could not be visualized clearly, this vein was detected by using color doppler flow. This distance could be measured for all patients.

To confirm reproduction, we used this method on 5 healthy volunteers, measuring each parameter twice, 3 days after the first investigation. Moreover, two experienced sonographers performed US on these volunteers to confirm reproduction. For serial observation of visceral fat, the progress of the 5 patients with diabetes mellitus who had a BMI of over 30 was followed using both US and CT at 0,3 and 6 months under diet therapy. These patients underwent diet therapy and physical exercise.

\section{CT scanning}

CT was performed in the supine position, using a LightSpeed Ultra 16 (GE Medical Systems, Milwaukee, WI). The interval of the single scan was $10 \mathrm{~mm}$ in the abdominal area. The visceral fat volume was calculated at the fourth lumbar vertebra according to the previous reports (9-12).

\section{Statistical analysis}

Student's $t$-test for unpaired samples was used to analyze the significance of difference in biochemical measurements between the visceral fat type group and the non-visceral fat 


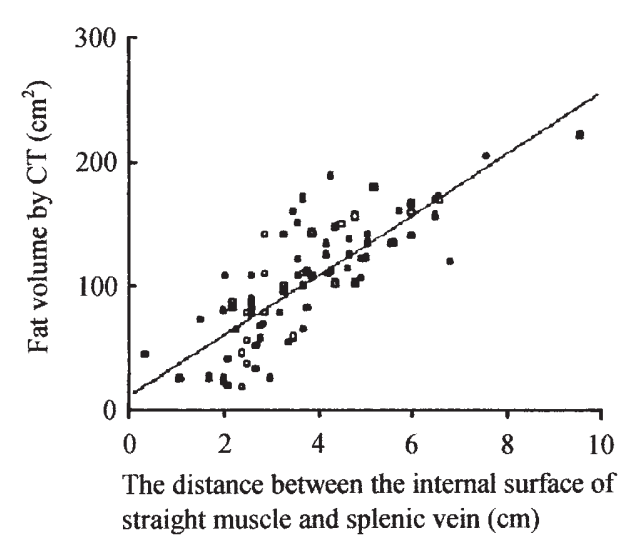

A

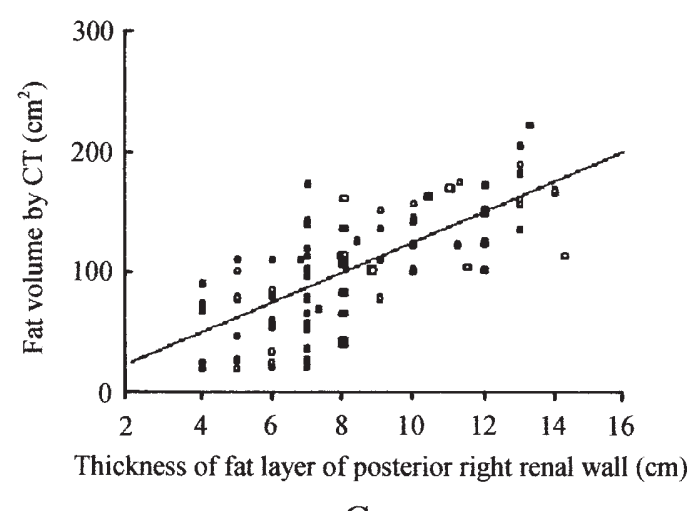

$\mathrm{C}$

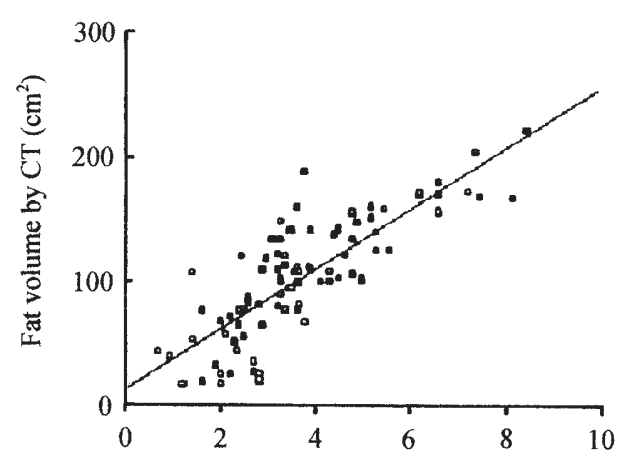

The distance between the internal surface of straight muscle posterior wall of aorta on umbilicus $(\mathrm{cm})$

B

Figure 2. Relation between parameters of US and fat volume measured by CT. A) The distance between the internal surface of the abdominal muscle and the splenic vein $(r=0.801, p<0.0001), B)$ the distance between the internal surface of the abdominal muscle and the posterior wall of aorta on umbilicus $(r=0.813, p<0.0001), C)$ the thickness of the fat layer of the posterior right renal wall $(\mathrm{r}=0.719, \mathrm{p}<0.0001)$.

type group. Pearson's correlation coefficients were calculated to assess the association among the factors using US, and the visceral fat volume was calculated using CT. Multivariate analysis with a stepwise selection process was used. $\mathrm{P}$ values of less than 0.05 were considered significant. Data analysis was performed using the computer program SPSS, Version 10.0 (SPSS Inc., Chicago, USA).

\section{Results}

Two different experienced sonographers measured these parameters of visceral fat for five healthy volunteers. The distance between the internal surface of the abdominal muscle and the splenic vein and the visceral fat volume was $3.58 \pm 0.60 \mathrm{~cm}$ and $3.50 \pm 0.62 \mathrm{~cm}$, respectively $(\mathrm{p}=0.841)$. The internal surface of the abdominal muscle and the posterior wall of the aorta on the umbilicus was $3.20 \pm 1.28 \mathrm{~cm}$ and $3.12 \pm 1.16 \mathrm{~cm}$, respectively $(\mathrm{p}=0.92)$. The thickness of the fat layer of the posterior right renal wall was $5.80 \pm 2.68 \mathrm{~mm}$ and $5.80 \pm 2.49 \mathrm{~mm}$, respectively $(\mathrm{p}=0.94)$. There were no

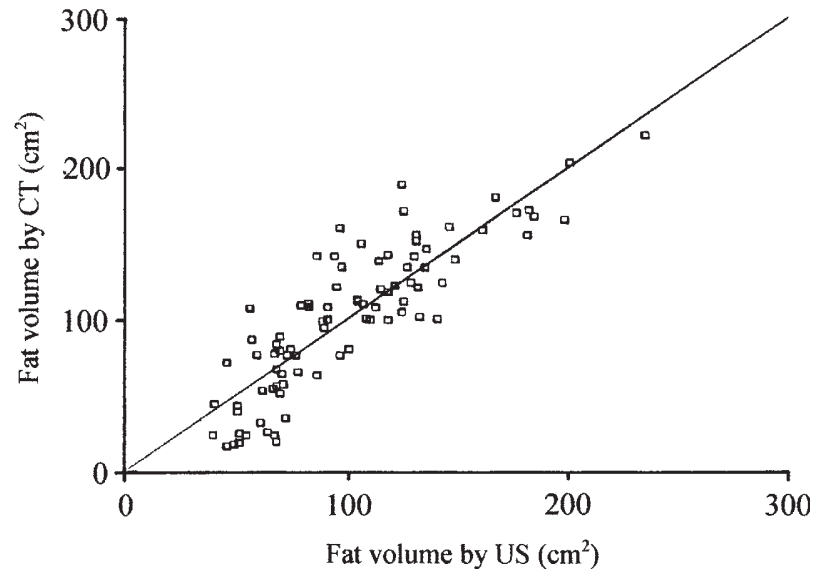

Figure 3. Relation between visceral fat volume by US and by CT. A good correlation between visceral fat volume by US and by $\mathrm{CT}$ was seen $(\mathrm{r}=0.860, \mathrm{p}<0.0001)$. 

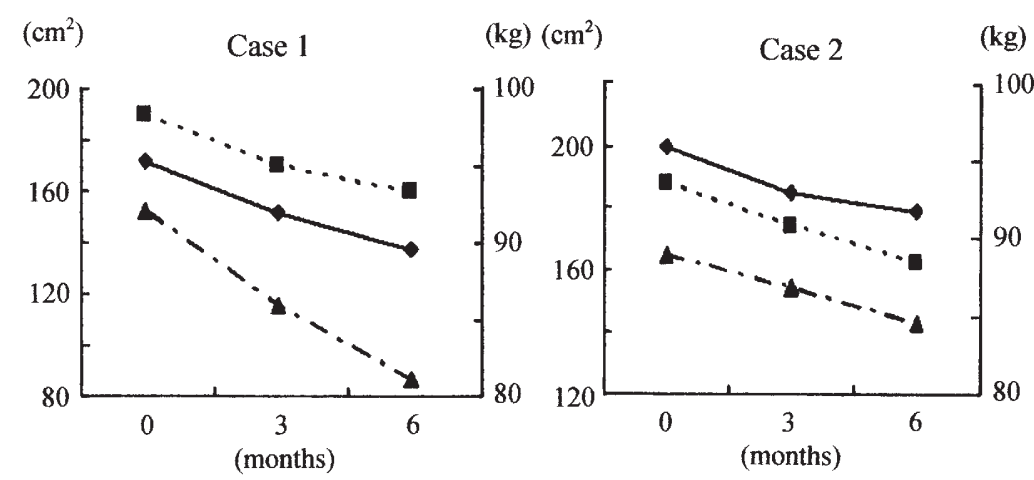

(kg) $\left(\mathrm{cm}^{2}\right)$

Case 3

(kg)

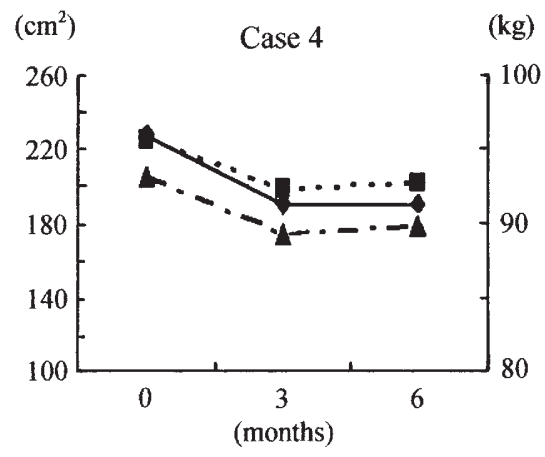

$\left(\mathrm{cm}^{2}\right)$

Case 5

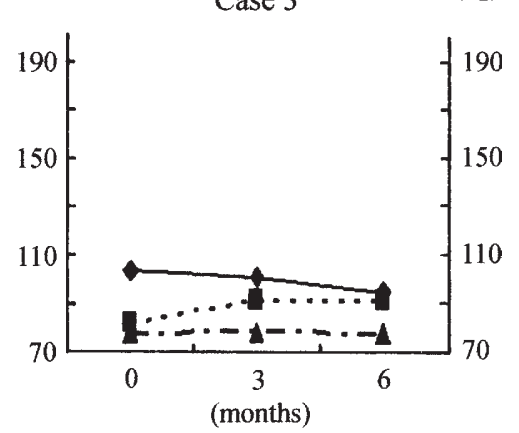

190

50

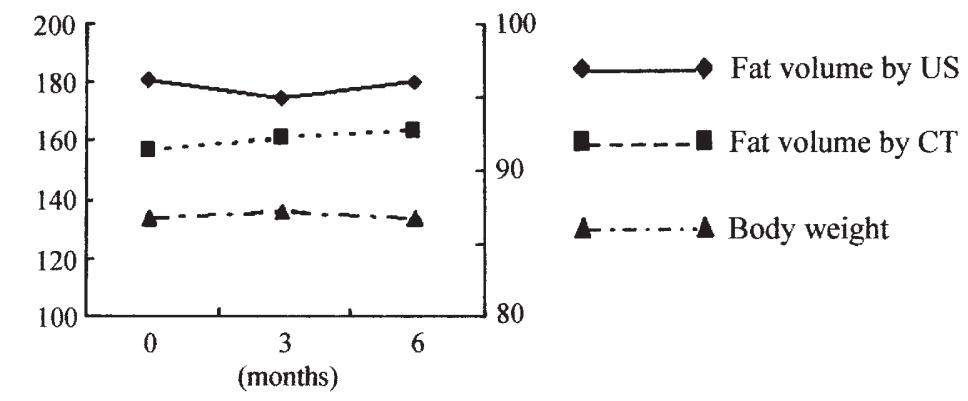

Figure 4. Serial observation in 5 patients who had a BMI of more than 30. In Cases 1 and 2 , the fat volume using both CT and US decreased, and the body weight also decreased. When there were no changes in body weight, the calculated fat volumes were not decreased.

significant differences for in these parameters between the two experienced sonographers.

Multivariate analysis was performed to obtain the significant contributing factors to predict the visceral fat volume. In the investigation of the relation between the factors measured using US and the visceral fat volume calculated using $\mathrm{CT}$, there was a significant correlation between the distance between the internal surface of the abdominal muscle and the splenic vein and the visceral fat volume $(r=0.801, p<0.0001$, Fig. 2A). The internal surface of the abdominal muscle and the posterior wall of the aorta on the umbilicus $(\mathrm{r}=0.813$, $\mathrm{p}<0.0001$, Fig. 2B) and the thickness of the fat layer of the posterior right renal wall $(r=0.719, p<0.0001$, Fig. $2 \mathrm{C})$ also significantly correlated with the visceral fat volume.

Using multivariate analysis, the equation for the visceral fat volume was calculated as follows: [visceral fat volume]= $-9.008+1.191 \times[$ The distance between the internal surface of the straight muscle and the splenic vein $(\mathrm{mm})]+0.987 \times[$ The distance between the internal surface of the straight muscle and the posterior wall of the aorta on the umbilicus (mm)] $+3.644 \times$ [the thickness of the fat layer of the posterior right renal wall with a significant correlation $(\mathrm{mm})]$. There was a strong correlation between the visceral fat volume calculated from the above equation and the volume by $\mathrm{CT}$ described $(r=0.860, p<0.0001$, Fig. 3). In 5 volunteers, there was no significant difference between the fat volumes at the first US $\left(86.4 \pm 25.3 \mathrm{~cm}^{2}\right)$ and the second US $\left(84.7 \pm 24.9 \mathrm{~cm}^{2}, \mathrm{p}=\right.$
$0.915)$.

In 5 patients who had a BMI of more than 30, we prospectively performed CT and US for 3 times. In Cases 1 and 2, the fat volume using both CT and US decreased, and the also body weight decreased. In the subjects who showed no change in body weight, the calculated fat volumes did not decrease (Fig. 4).

Using the visceral fat volume calculated by US, we compared the biochemical markers in 36 patients having a BMI of more than 25. Of 36 patients who had a BMI of more than 25 , the rate of non-visceral fat type patients was $33 \%$. Fasting plasma glucose $(\mathrm{p}<0.05)$, total cholesterol $(\mathrm{p}<0.05)$, LDL-cholesterol $(\mathrm{p}<0.01)$, free fatty acid $(\mathrm{p}<0.01)$, leptin $(\mathrm{p}<0.01)$ and HOMA-R $(\mathrm{p}<0.01)$ were increased in the visceral fat group (Table 1).

\section{Discussion}

Excessive accumulation of adipose tissue causes obesity. There have been many reports on the significance of visceral fat volume (4-6). Some groups report that obese people with more than $100 \mathrm{~cm}^{2}$ of visceral fat volume have a higher complication rate of diabetes mellitus and coronary disease ( 7 , 23). Therefore, a visceral fat volume of over $100 \mathrm{~cm}^{2}$ is widely accepted in diagnosing visceral fat obesity. However, if only weight or BMI are measured, this is not diagnosed as visceral fat type. Of the patients who had a BMI of more 
HiROOKA et al

Table 1. Comparison of Biochemical Markers

\begin{tabular}{|c|c|c|c|}
\hline & Non visceral fat group & Visceral fat group & \\
\hline $\mathrm{N}$ & 12 & 24 & \\
\hline Age (years) & $55.3 \pm 17.3$ & $55.7 \pm 15.2$ & \\
\hline Body mass index $\left(\mathrm{kg} / \mathrm{cm}^{2}\right)$ & $27.2 \pm 1.41$ & $28.2 \pm 3.3$ & \\
\hline Fasting plasma glucose (mg/dl) & $94.8 \pm 9.50$ & $128.6 \pm 43.1$ & $*$ \\
\hline Hemoglobin A1c (\%) & $5.3 \pm 1.3$ & $6.1 \pm 1.2$ & \\
\hline Total cholesterol (mg/dl) & $177.7 \pm 36.7$ & $207.3 \pm 31.8$ & $*$ \\
\hline Triglyceride (mg/dl) & $130.1 \pm 44.2$ & $154.9 \pm 51.1$ & \\
\hline High density lipoprotein (mg/dl) & $56.3 \pm 16.2$ & $47.6 \pm 14.0$ & \\
\hline Low density lipoprotein $(\mathrm{mg} / \mathrm{dl})$ & $96.5 \pm 37.0$ & $129.6 \pm 28.7$ & $* *$ \\
\hline Nonesterified fatty acid $(\mathrm{mEq} / \mathrm{l})$ & $0.34 \pm 0.23$ & $0.72 \pm 0.18$ & $* *$ \\
\hline Uric acid (mg/dl) & $5.7 \pm 1.7$ & $5.7 \pm 1.2$ & \\
\hline Alanine transferase (IU/l) & $42.3 \pm 24.9$ & $73.9 \pm 56.9$ & \\
\hline Leptin (ng/ml) & $12.8 \pm 4.40$ & $19.9 \pm 5.65$ & $* *$ \\
\hline Homeostasis model assessment & $2.14 \pm 1.14$ & $5.76 \pm 2.71$ & $* *$ \\
\hline
\end{tabular}

$* \mathrm{p}<0.05, * *<0.01$

than 25 which had been thought to be the visceral fat type (7), the rate of non-visceral fat type patients was $33 \%$. This suggests that BMI is not a useful method for detecting the visceral fat type. So in order to diagnose visceral fat type, it is better to perform abdominal images (i.e. CT, MRI, or US). Both CT and US have been widely used and have been effective in many fields. CT, however exposes patients to ionizing radiation, whereas US does not expose the patient to such radiation. There have been no methods for easy and accurate diagnosis of visceral fat obesity. However, we have developed a new, simple and accurate method of measuring visceral fat volume using US.

We have noted that the splenic vein is unclear in obese people due to the accumulation of visceral fat. So we focused on the distance between the internal surface of the abdominal muscle and the splenic vein (Fig. 1a), which to date has not been considered as a factor. Other distances, which have been studied previously, were also measured (20-22). The distance between the internal surface of the abdominal muscle and the splenic vein was as useful as the distance between the internal surface of the abdominal muscle and the posterior wall of aorta on the umbilicus and the thickness of the fat layer of the posterior right renal wall. In those reports (20-22), only a single parameter was used. Thus, to obtain a stronger correlation with the visceral fat volume using CT, we produced a novel equation using multivariate analysis. We measured each parameter twice in 5 healthy volunteers, and found that the role of US is important, there being less difference between the 2 measurements. Moreover, 2 experienced sonographers measured the parameter, and calculated fat volumes that were not significantly different. Thus, this method has a good reproduction. In the following patients with decreasing body weight and fat volume according to $\mathrm{CT}$, the fat volume using US was also decreased. Thus, the method that uses US is useful to evaluate the efficacy of diet therapy and physical exercise.
In addition in the 36 patients in the present study who had a BMI of more than 25 (which had been thought as visceral fat type) (7), the rate of non-visceral fat type patients was $33 \%$. In the visceral fat type group, HOMA-R, fasting plasma glucose, total cholesterol, and LDL-cholesterol were significantly high. Obese people in the visceral fat type group show insulin resistance, and are a high-risk group for metabolic disorders because free fatty acids and leptin were significantly high. Thus, the patients who had a BMI of more than 25 should be divided into 2 groups based on ultrasonography.

In conclusion, the measurement of visceral fat volume using US could calculate as effectively as using CT. This method should be practiced in clinics due to its low cost, no side effects, and technical suitability.

Acknowledgements: We wish to thank Dr. Takeshi Kubo (Gynecology, Saiseikai Ryugasaki Hospital, Japan) for performing the statistical analysis.

\section{References}

1) Reaven GM. Banting lecture. Role of insulin resistance in human disease. Diabetes 37: 1595-1607, 1988.

2) Kaplan NM. The deadly quartet: upper-body obesity, glucose intolerance, hypertriglyceridemia, and hypertension. Arch Intern Med 149: 1514-1520, 1989.

3) Defronzo RA, Ferrannini E. Insulin resistance: A multifaceted syndrome responsible for NIDDM, obesity, hypertension, dyslipemia, and atherosclerotic cardiovascular disease. Diabetes Care 14: 173-194, 1991.

4) Fujioka S, Matsuzawa $Y$, Tokunaga $T$, Tarui S. Contribution of intraabdominal fat accumulation to the impairment of glucose and lipid metabolism in human obesity. Metabolism 36: 54-59, 1987.

5) Nakamura T, Tokunaga K, Shimomura I, et al. Contribution of visceral fat accumulation to the development of coronary artery disease in nonobese men. Atheroscrelosis 107: 239-246, 1994.

6) Matsuzawa Y. Pathophysiology and molecular mechanism of visceral fat syndrome. Diabetes Metab Rev 13: 3-13, 1997.

7) The Examination Committee of Criteria for "Obesity disease" in Japan; 


\section{The Measurement of Visceral Fat by Ultrasonography}

Japan Society for the Study of Obesity. New criteria for 'obesity disease' in Japan. Circ J 66: 987-992, 2002

8) Han TS, van Leer EM, Seidell JC, Lean MEJ. Waist circumference action levels in the identification of cardiovascular risk factors: prevalence study in a random sample. BMJ 311: 1401-1405, 1995.

9) Tokunaga K, Matsuzawa Y, Ishikawa K, Tarui S. A novel technique for the determination of body fat by computed tomography. Int J Obes 7: 437-445, 1983 .

10) Busetto L, Baggio MB, Zurlo F, Carraro R, Digito M, Enzi G. Assessment of abdominal fat distribution in obese patients: anthropometry versus computerized tomography. Int J Obes Relat Metab Disord 16: 731-736, 1992.

11) Stallone DD, Stunkard AJ, Wadden TA, Foster GD, Boorstein J, Arger P. Weight loss and body fat distribution: a feasibility study using computed tomography. Int J Obes 15: 775-780, 1991.

12) Dixon AK. Abdominal fat assessed by computed tomography: sex difference in distribution. Clin Radiol 34: 189-191, 1983.

13) de Ridder CM, de Boer RW, Seidell JC, et al. Body fat distribution in pubertal girls quantified by magnetic resonance imaging. Int $\mathrm{J}$ Obes 16: 443-449, 1992.

14) Staten MA, Totty WG, Kohrt WM. Measurement of fat distribution by magnetic resonance imaging. Invest Radiol 24: 345-349, 1989.

15) Chan YL, Leung SS, Lam WW, Peng XH, Metreweli C. Body fat estimation in children by magnetic resonance imaging, bioelectrical impedance, skinfold and body mass index: a pilot study. J Paediatr Child Health 34: 22-28, 1998.

16) Caprio S, Hyman LD, McCarthy S, Lange R, Bronson M, Tamborlane
WV. Fat distribution and cardiovascular risk factors in obese adolescent girls: importance of the intraabdominal fat depot. Am J Clin Nutr 64: 12-17,1996.

17) Owens S, Gutin B, Ferguson M, Allison J, Karp W, Le NA. Visceral adipose tissue and cardiovascular risk factors in obese children. J Pediatr 133: 41-45,1998.

18) Leite CC, Wajchenberg BL, Radominski R, Matsuda D, Cerri GG, Halpern A. Intra-abdominal thickness by ultrasonography to predict risk factors for cardiovascular disease and its correlation with anthropometric measurements. Metabolism 51: 1034-1040, 2002.

19) Pontiroli AE, Pizzocri P, Giacomelli M, et al. Ultrasound measurement of visceral and subcutaneous fat in morbidly obese patients before and after laparoscopic adjustable gastric banding: comparison with computerized tomography and with anthropometric measurements. Obes Surg 12: 648-651, 2002.

20) Armellini F, Zamboni M, Castelli S, et al. Measured and predicted total and visceral adipose tissue in women. Correlations with metabolic parameters. Int J Obes Relat Metab Disord 18: 641-647, 1994.

21) Armellini F, Zamboni M, Rigo L, et al. The contribution of sonography to the measurement of intra-abdominal fat. J Clin Ultrasound 18: 563$567,1990$.

22) Suzuki R, Watanabe S, Hirai $Y$, et al. Abdominal wall fat index, estimated by ultrasonography, for assessment of the ratio of visceral fat to subcutaneous fat in the abdomen. Am J Med 95: 309-314, 1993.

23) Despres JP, Lamarche B. Effects of diet and physical activity of adiposity and body fat distribution: Implications for the prevention of cardiovascular disease. Nutr Res Rev 6: 137-159, 1993. 